\title{
Compact and powerful THz source investigation on laser plasma wakefield injector and dielectric lined structure
}

\author{
L. Shi $\odot,{ }^{*}$ S. L. Johnson, ${ }^{\dagger}$ and S. Reiche \\ Paul Scherrer Institut, 5232 Villigen, Switzerland \\ (Received 22 August 2019; published 3 January 2020)
}

\begin{abstract}
The advancement of the techniques of producing electromagnetic radiation in the terahertz range (1-20 THz) attracts a wide range of potential applications and the quest for powerful THz sources is everincreasing. The means to generate $\mathrm{THz}$ is an active research field and the energy per pulse is limited due to breakdown of the medium used. In this paper, we propose a compact yet powerful narrowband $\mathrm{THz}$ source based on two pillars of active research in the field of novel accelerators: laser plasma wakefield acceleration and high gradient dielectric lined structures. The laser plasma wakefield (LPW) injector is used to provide the relativistic electron bunches to an interaction tube, a dielectric lined waveguide (DLW), in order to generate THz. Due to the high gradient nature of both devices, the combination ends up with a very compact solution for $\mathrm{mJ}$ scale $\mathrm{THz}$ pulse generation. Potentially these pulses can be used as drivers for nonlinear phenomena in condensed matter, which can be probed using ultrashort X-ray pulses at the same free electron laser facility.
\end{abstract}

DOI: 10.1103/PhysRevAccelBeams.23.014701

\section{INTRODUCTION}

Electromagnetic radiation in the terahertz $(\mathrm{THz})$ range, which lies between approximately 1 and $20 \mathrm{THz}$, can interact with matter in several remarkable ways, offering unique opportunities both as a spectroscopic probe or, at higher intensities, as a means to potentially engineer new states of matter [1]. One key advantage of THz-frequency radiation is tied to its low photon energy $(h \nu=4.1 \mathrm{meV}$ for $\nu=1 \mathrm{THz}$ ). In many biological, chemical, and physical systems, THz interactions are nonionizing and can often be tuned to specific material resonances [2]. One particularly exciting possibility is to combine intense $\mathrm{THz}$ radiation with ultrashort and intense x-ray pulses from a free electron laser facility, e.g., SwissFEL (the free electron laser in Switzerland) [3], which can measure the dynamics of charge, spin and ions in response to resonant $\mathrm{THz}$ excitation of selected resonances [4]. Reference [5] provides a general overview of THz-based experiments and their road map.

Due to the appealing THz-matter interaction characteristics aforementioned and its own scientific value, many methods have been proposed to generate $\mathrm{THz}$ [6], including

\footnotetext{
*liangliang.shi@psi.ch

Also at Institute for Quantum Electronics, Eidgenossische Technishe Hochschule (ETH) Zürich, 8093 Zürich, Switzerland.

Published by the American Physical Society under the terms of the Creative Commons Attribution 4.0 International license. Further distribution of this work must maintain attribution to the author(s) and the published article's title, journal citation, and DOI.
}

but not limited to the methods based on solid state oscillators [7], plasma dipole oscillators [8], quantum cascaded laser [9], laser pumped solid state devices [10] and electron beam accelerators $[11,12]$ etc. However, most of these methods generally suffer from low energy per pulse. In this paper, we report on a $\mathrm{THz}$ radiator based on an electron beam and dielectric lined waveguide (DLW), a structure similar to that reported in Ref. [11]. We aim to investigate the suitability of this method for the high energy $\mathrm{THz}$ pulse generation for pump-probe experiments. This constrains the DLW structure to be small enough transversely to provide $\mathrm{THz}$ pulses in $1-20 \mathrm{THz}$ compared with the predominantly published experimental results, which are normally around hundred GHz. The actual frequency of interest depends of course on the target users and the radiator should also be able to tune over a certain range.

The relativistic electron beam needed to drive the DLW structure is normally from an accelerator beam line such as Refs. $[11,13]$. In order to provide an electron beam with hundred $\mathrm{MeV}$, current rf accelerating technology requires an electron injector and an accelerator of a few tens of meters irrespective of normal conducting or superconducting radio frequency cavities [14-17]. Novel acceleration schemes like plasma wakefield accelerators promise a GV/ $\mathrm{cm}$ accelerating gradient. These can provide kA electron beam currents with $\mu \mathrm{m}$ transverse sizes ideal for $\mathrm{THz}$ generation. In this paper, we exclusively investigate the use of these electron bunches from a laser plasma wakefield accelerator, which works essentially as an injector in our case. The acceleration concept was first proposed by Tajima and Downson in 1979 [18] and the experimental 
demonstration of monoenergetic electron beams are blooming in the 2000s [19-21], which benefited directly from the high power laser system that can drive plasma wakefields based on the chirped pulse amplification technique [22]. The electron beam out of such an injector normally features percent level of energy spread and mrad divergence. As we will see in the rest of the paper, the $\mathrm{THz}$ generation will be insensitive to the energy spread for a relativistic electron beam. A certain tunability can also be achieved with nonrelativistic electron beam [23]. The high initial divergence still has to controlled, which in fact limits the interaction length to a few centimeters without any focusing magnets. Provided the external focusing magnets are used to compensate the divergence, the interaction length can be increased accordingly. The length partially dictates the bandwidth of the produced $\mathrm{THz}$ pulses. The investigation was intended for a possible narrowband (less than $10 \%) \mathrm{THz}$ source at the SwissFEL free electron laser facility.

SwissFEL is based on normal conducting $\mathrm{S}$ and $\mathrm{C}$ band rf technology for the electron beam acceleration and it is designed to provide soft and hard $\mathrm{x}$-rays within the range of $0.7-5 \mathrm{~nm}$ and $0.1-0.7 \mathrm{~nm}$ respectively of beamlines Athos and Aramis [3]. There is general interest in using $\mathrm{THz}$ pulses in combination with the ultrashort $\mathrm{x}$-rays. We have proposed one possible solution by using the spent electron beam from Aramis to generate $\mathrm{THz}$ for the users at Athos [13]. This paper alternatively proposes the use of electrons from a laser plasma injector to produce THz. This will enable a flexibility of the deployment of the THz source in comparison with a fixed electron source from SwissFEL.

The paper is organized as follows: in Sec. II, we report the operational principle of laser plasma wakefield (LPW) injector and the dielectric lined waveguide. The capture and transport of the relativistic electron bunch from LPW to the interaction DLW is investigated in Sec. III. The interaction between the electron beam and the DLW is summarized in Sec. IV. Some potential applications are discussed in Sec. V. In the last section, we conclude the paper and discuss potential future work.

\section{OPERATIONAL PRINCIPLE OF THE THz GENERATION}

The generation of the $\mathrm{THz}$ pulses relies on wakefield generation when an electron beam traverses a dielectric lined waveguide and the electron beams are normally from conventional rf accelerators, as reported in many published experimental results $[11,24-26]$. The relativistic electron bunches can also be generated from a laser plasma injector, which is used to drive dielectric lined structures at a later stage. The key motivation of combining the two is the fact that the $\mathrm{THz}$ generation process is insensitive to the energy spread of the electron beam coming out of the laser plasma accelerating process. We discuss the generation of the relativistic electron bunches in subsection II A and the working principle of the dielectric structures and the interaction in subsection II B 1 separately. The capture and transportation of the electron bunches to the interaction dielectric structures will be tackled in Sec. III.

\section{A. Relativistic electron bunch generation}

The relativistic electrons can be generated via a dedicated rf injector as in many free electron laser facilities, e.g., SwissFEL. The electron bunch, however, is limited partially due to space charge effects and has to be accelerated as quickly as possible to become relativistic via rf accelerating cavities. The rf accelerating cavities routinely used in running accelerators are limited to be approximately several tens of $\mathrm{MV} / \mathrm{m}$ regardless of normal conducting or superconducting technologies. This would require a long section of acceleration to produce several hundred $\mathrm{MeV}$ beam and also necessitate the beam operation magnets for shorter bunches. The intensive $R \& D$ researches about high gradient structures are widely carried out worldwide. Among them, laser plasma acceleration attracts much attention due to the $\mathrm{GV} / \mathrm{cm}$ accelerating gradient demonstrated experimentally [19-21] and the ability to work as an injector as well.

The relativistic electron bunch generation relies on the nonlinear process of laser and plasma interaction [27]. When a laser pulse with sufficient strength traverses an underdense plasma, the quiver motion of electron becomes relativistic and the laser plasma interaction is nonlinear. The ambient electrons in the plasma are pushed away by the ponderamotive force from the propagating laser pulse and bubble region with only ions is formed [27]. These stray electrons potentially can be trapped in the bubble and form a electron bunch to be accelerated.

The accelerating bubble cavity has strong dependence on both the laser and plasma parameters. Therefore, it is vital to form the plasma in a capillary so that the interaction plasma profile is reproducible. Tailoring the cavity shape with laser and plasma parameters is an active research field to better control the electron beam quality during acceleration. The normalized laser strength has explicit dependence on its wavelength $\lambda$ and intensity $I_{0}$,

$$
a_{0} \simeq 8.54 \times 10^{-10}[\lambda(\mu \mathrm{m})] \sqrt{I_{0}\left(\mathrm{~W} / \mathrm{cm}^{2}\right)} .
$$

The requirement of $a_{0}>1$ dictates the laser intensity grater than $10^{18} \mathrm{~W} / \mathrm{cm}^{2}$ for $800 \mathrm{~nm}$ laser, which can be routinely obtained based on the CPA technique with a commercially available laser system. The laser peak power used in the published experimental results varies from several TW to PW with a pulse duration in a few tens of fs. The accelerating gradient of these accelerating bubble structure can be estimated with $E_{0}(\mathrm{~V} / \mathrm{m}) \simeq 96 \sqrt{n_{0}\left(\mathrm{~cm}^{-3}\right)}$ and the ambient electron density $n_{0}$ is normally on the order of $10^{17}-10^{19} / \mathrm{cm}^{3}$, which supports $\mathrm{GV} / \mathrm{cm}$ accelerating gradient. A hundred $\mathrm{MeV}$ electron beam will require only a few 
$\mathrm{mm}$ interaction length, which is feasible in a single stage acceleration and is well below the cutting-edge LPWs.

Short bunches are readily available from LPWs and the wavelength of the accelerating wakefield wave is,

$$
\lambda_{p}(\mu \mathrm{m}) \simeq 3.3 \times 10^{10} / \sqrt{n_{0}\left(\mathrm{~cm}^{-3}\right)}
$$

where $\lambda_{p} \simeq 3.3-33 \mu \mathrm{m}$ for $n_{0}=10^{19}-10^{17} \mathrm{~cm}^{-3}$. If the accelerated electron bunch takes only a fraction of this length, a short bunch in a few $\mu \mathrm{m}$ is directly accessible and this conforms to the published results.

Because these trapped electrons are quasisynchronized with the wakefield, they can be accelerated over some distance before the electron overtakes due to dephasing [27], which limits the interaction length over a single stage. The electron beam dynamics inside the bubble and the dynamics of the bubble have strong influence on the final beam quality when it exits the plasma interaction region. The beam normally has $\mu \mathrm{m}$ level transverse size, percent level of energy spread and mrad scale divergence. The relative large energy spread and mrad divergence impede the transport of the beam over long distance with conventional magnetic beam line as we will discuss in Sec. III. Many schemes have been invented to improve the beam quality by changing the laser or the plasma parameters [28]. Several schemes have been proposed for better control of the electron beam quality by methods such as self-trapping [29], ionization injection [30], negative density profile [31]. We summarize some beam parameters for the later study based on the published experimental results. Because of the vast body of researches worldwide and its rapid development, it is difficult to make an exhaustive list. The parameters are rather intended to provide an approximate parameter space for the first high energy $\mathrm{THz}$ pulse generation. They are obviously not the best beam quality achieved but rather conservative in order to relax the requirements on the laser and plasma source so that the proof of principle experiments can be carried out in the near future. Any further improvements, especially the beam divergence, will ease the overall design and improve the $\mathrm{THz}$ pulses in general. The desired electron beam parameters are summarized in Table I.

The self trapping based on wave breaking works in 100$1000 \mathrm{MeV}$ range [32]. Although high quality $\mathrm{GeV}$ beam are accessible experimentally, we here consider in the next section to use $100 \mathrm{pC}$ charge and $350 \mathrm{MeV}$ beam energy without concern about the energy spread. The laser pulse required in order to generate such an electron bunch would be with sub 100 TW peak power and a few tens of fs FWHM (full width half maximum) duration. The electron beam kinetic energy is $35 \mathrm{~mJ}$ in this case and a radiation pulse energy of $17.5 \mathrm{~mJ}$ can be obtained even with a moderate conversion efficiency of 50\% upon a successful transport of the electron bunches through DLW, which is
TABLE I. Beam parameters from LPA.

\begin{tabular}{lccc}
\hline \hline Parameter & Symbol & Value & Unit \\
\hline Beam Energy & $\gamma_{0} m c^{2}$ & $\leq 1000$ & $\mathrm{MeV}$ \\
RMS Energy spread & $\sigma_{\delta}$ & $\leq 10 \%$ & $/$ \\
RMS Beam size & $\sigma_{x, y}$ & $\leq 10$ & $\mu \mathrm{m}$ \\
RMS Beam Divergence & $\sigma_{x, y}^{\prime}$ & $\leq 5$ & $\mathrm{mrad}$ \\
Charge & $Q$ & $\leq 100$ & $\mathrm{pC}$ \\
RMS Bunch Length & $L_{s}$ & $\leq 20$ & $f s$ \\
\hline \hline
\end{tabular}

the topic in Sec. III. We will use this as a reference case whenever appropriate.

\section{B. Dielectric lined waveguide and wakefield}

The quest for a high-gradient accelerating structure, the same motivation for the development of plasma wakefield cavities, triggered another research area: dielectric lined waveguide (DLW). The DLW was first considered for accelerating structures decades ago [33-39] and later has spun off as high power electromagnetic radiation sources $[11,24,26,40,41]$. The theoretical analysis of the mode structures and the wakefield theory are discussed thoroughly in pioneering works $[33,34,42-44]$ etc. We will first consider a basic DLW structure as a reference case. The wakefield is reviewed in the second part of this section.

\section{1. $D L W$}

A circular dielectric lined waveguide is considered here and the section view along the rotation axis $z$ is shown in Fig. 1. In principle, the waveguide can be of any cross section but a translational symmetry along $z$ is imposed in order to make the analytical analysis feasible. The waveguide with other cross geometries can be analyzed with conformal mapping [45].

The DLW is formed with a vacuum channel of radius $a$ for the transport of the electron bunch and a surrounding dielectric layer characterized with permittivity $\varepsilon$ with a layer thickness of $b-a$. The outside of the dielectric layer is coated with metal, which confines the electromagnetic field inside the DLW. The confined field is excited when a electron bunch traverses the DLW.

The DLW is characterized with four parameters, inner radius $a$, outer radius $b$, dielectric layer with permittivity $\varepsilon$ and the length of the structure $L$. As we will see later, in order to generate $\mathrm{THz}$ between $1-20 \mathrm{THz}$, the transverse size of the structure is limited to a few hundred $\mu \mathrm{m}$ and the dielectric material considered here is quartz with $\varepsilon=3.8$ for this paper. The length of the structure varies from a few $\mathrm{cm}$ to a few tens $\mathrm{cm}$ in order to generate $\mathrm{mJ}$ pulses and this length also determines the number of cycles of the THz pulses inside a DLW, which is linked with the pulse bandwidth. A modification of the basic structure is favorable in some cases when one needs to intensify the generated $\mathrm{THz}$ pulses or to make 
some special manipulation on the THz pulses, e.g., tapering in $a$ for the chirped THz generation [46].

\section{Eigenmodes and wakefields of a DLW}

In a DLW, the electromagnetic field, which is transversely bounded forming a certain field distribution oscillating at a specific frequency, is called mode. The mode can travel along the $z$ direction as in Fig. 1. Following the name convention and methods used in, e.g., [34,42,43], the transverse magnetic (TM) mode frequency can be obtained by solving a dispersion relation as shown in Fig. 2 .

The intersections between light line $\left(\omega=k_{z} c \beta\right)$ and the dispersion curve define the modes $\mathrm{TM}_{01-6}$ frequencies for $\beta=1$ and 0.99 . The two almost indistinguishable light lines in Fig. 2 indicate that the mode frequencies are approximately independent of the normalized speed $\beta$ once the electron beam is relativistic. Qualitatively, for the maximum electron beam energy spread (10\%) that we consider here, the relative mode frequency detuning is less than $10^{-6}$. This small frequency variation is well within the bandwidth $(\leq 10 \%$ preferably $\leq 5 \%)$ required for various experiments. The mode frequencies depend only on the

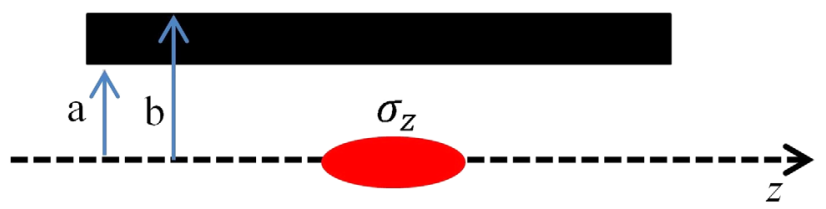

Electron bunch

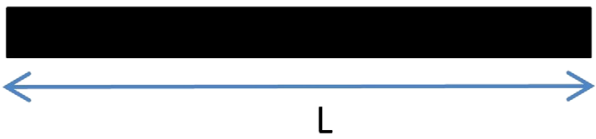

FIG. 1. DLW with relevant structure (not to scale).

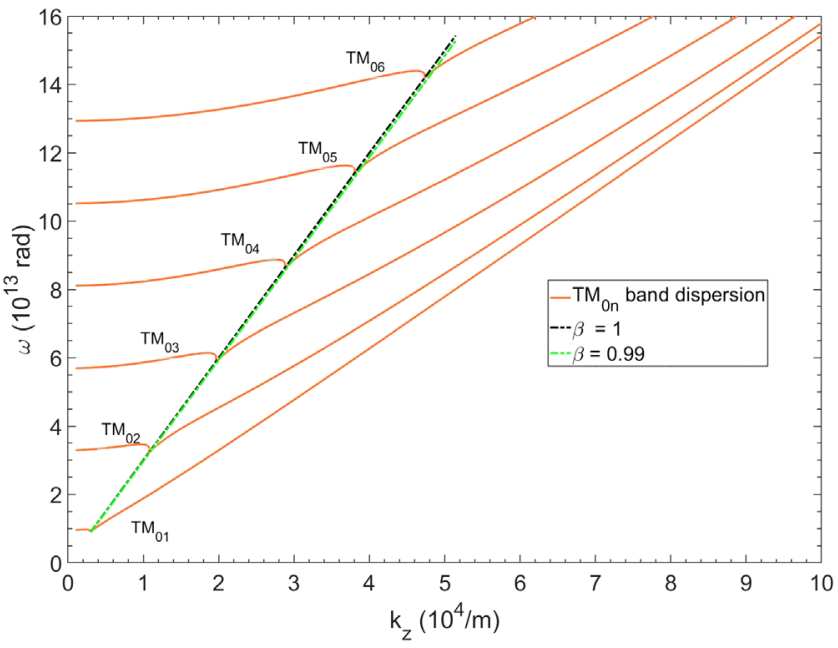

FIG. 2. Dispersion for the first six TM bands with $a=100 \mu \mathrm{m}$, $b=120 \mu \mathrm{m}$. Two light lines with $\beta=1$ and $\beta=0.99$ are shown and the intersection points determine the corresponding modes.

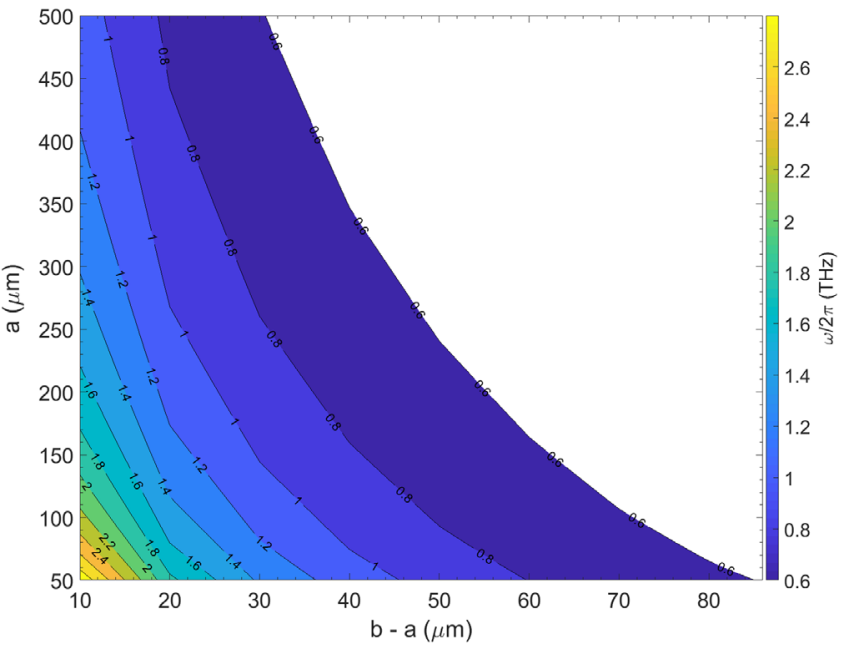

FIG. 3. $\mathrm{TM}_{01}$ mode frequency dependence on the DLW inner radius $a$ and dielectric thickness $b-a$.

parameters $a$ and $b$ for a fixed dielectric material. A summary of mode frequency map shows the dependence on both parameters in Fig. 3. In order to reach above $1 \mathrm{THz}$, the maximum $a$ can be close to $500 \mu \mathrm{m}$. The insensitivity to $\beta$ indicates the excitation process is insensitive to the energy spread of the electron beam. This allows us to use the electron beam with a large energy spread from laser plasma injector with less concern. The wakefield of a particular beam is studied via eigenmode expansion methods [43]. In the following sections, we will consider only the ultrarelativistic electron beam where we assume $\beta=1$. Therefore the associated wakefield formulation is independent of $\beta$ and the higher order effects of $\beta$ are mentioned whenever appropriate.

The longitudinal electrical field $E_{z}$ of the monopole modes is shown in Fig. 4, which accounts for the $\mathrm{THz}$ generation. It is effectively constant in the vacuum passage for the $\mathrm{TM}_{0 \mathrm{n}}$ modes. The excited monopole modes therefore have no dependence on the beam transverse distribution but only on the longitudinal one, which ensures a stable $\mathrm{THz}$ intensity.

The electron beam excited field can be expanded in terms of modes as in [43]. The energy loss $U_{n}$ to a given mode is related to the loss factor $\kappa_{n}$ and the charge $q$ by $U_{n}=\kappa_{n} q^{2}$. The total energy loss $U_{\text {tot }}$ due to a single point charge $q$ is

$$
U_{\mathrm{tot}}=\frac{q^{2}}{2 \pi \varepsilon_{0}} \frac{L}{a^{2}}=q^{2} \sum_{n=1}^{\infty} \kappa_{n} .
$$

As an example, the mode power and the total power by summing up consecutive modes is shown in Fig. 5. A first few modes will cover the frequency range between 1 and $20 \mathrm{THz}$ and account for most of the energy loss from a bunch.

A parametric study of the pulse energy is shown in Fig. 6. In order to generate $\mathrm{mJ}$ scale pulse, there is a constraint on the length of the structure and the inner radius. Higher pulse 


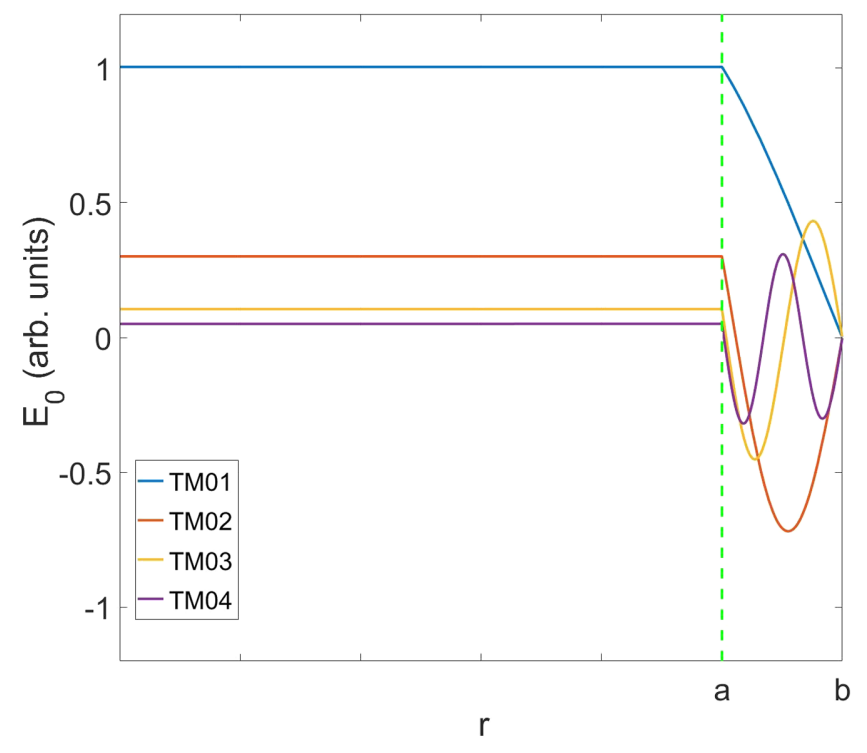

FIG. 4. Transverse profile of the longitudinal field strength of the $\mathrm{TM}_{0 \mathrm{n}}$ modes.

energy requires smaller $a$ and longer $L$. For example, in order to generate $1 \mathrm{~mJ}$ THz pulse, a DLW with $300 \mu$ m inner radius would require the transport of $100 \mathrm{pC}$ over $50 \mathrm{~cm}$. This distance is already comparably long due to the achievable beam capture transport optics as we will see in the next section.

Because the longitudinal and transverse wakefield scales with $1 / a^{2}$ and $1 / a^{3}$ respectively, the field gradient can be quite high for small aperture $a$. The uniform distribution of the longitudinal electrical field makes the vacuum and dielectric material interface vulnerable. In our case, it is on the order of $2 \mathrm{GV} / \mathrm{m}$, which is still below the breakdown

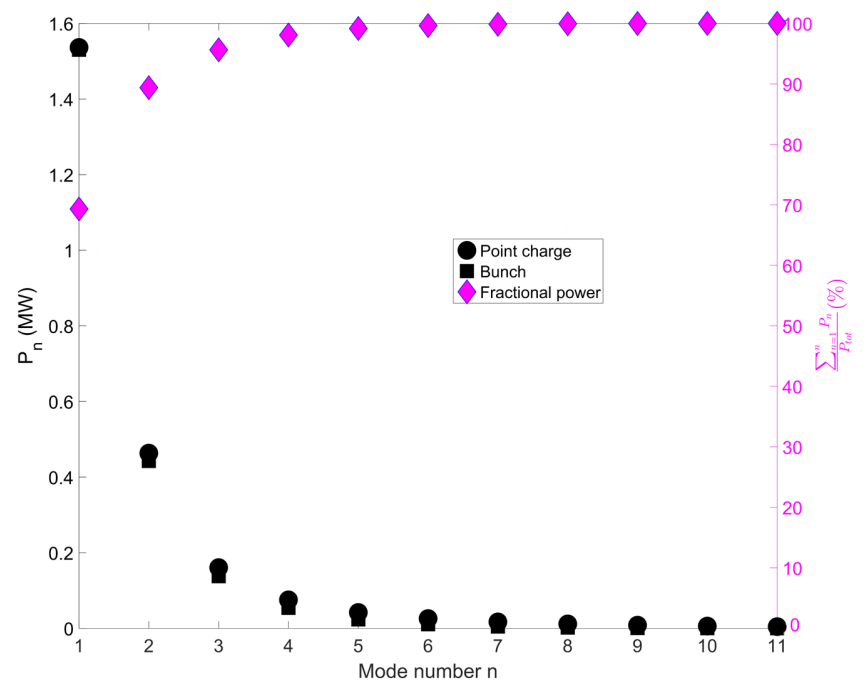

FIG. 5. Power spectrum for a charge of $100 \mathrm{pC}$ with $a=$ $148 \mu \mathrm{m}$ and $b=178 \mu \mathrm{m}$. We here considered point charge (black dots), a 20 fs long Gaussian bunch (black squares) and fractional power (magenta diamonds). limit $12 \mathrm{GV} / \mathrm{m}$ shown experimentally [47]. The strong transverse wakefields also impose stringent requirements on beam transport inside a DLW.

\section{BEAM TRANSPORTATION}

Capture and transport of the electron beam without significant beam quality degradation from the plasma channel to the interaction DLW structure turns out to be quite challenging for the considered beam parameters. Inside the DLW, the beam should be able to travel without significant losses. Two basic designs can be conceived for our application: 1 . Place a DLW directly next to the plasma capillary; 2. Capture the electron beam from the plasma capillary via a set of magnets. In the first case, the interaction length of the DLW is limited by the initial divergence of the electron beam. For the latter, the length of the DLW is jointly defined by the transport line and the DLW itself. We will consider the two approaches in the next two subsections.

\section{A. Direct capture}

Due to the small interaction length required for a DLW with small inner radius $a$ as indicated in Fig. 6, a DLW can be placed directly next to the plasma exit. A schematic of the setup is shown in Fig. 7.

The ultrarelativistic electron bunch is produced in the plasma stage and is transported directly to the DLW stage. This allows a compact yet powerful $\mathrm{THz}$ generator within just a few $\mathrm{cm}$. Due to the mrad scale divergent electron beam, the beam will get lost quickly if the DLW is too long. For example, a DLW of $1 \mathrm{~cm}$ will limit the inner radius $a$ to $10 \mu \mathrm{m}$ for $1 \mathrm{mrad}$ beam, which can still grant a very high pulse energy due to the $\mathrm{GV} / \mathrm{m}$ decelerating gradient. Any improvement of the beam divergence definitely favors a longer DLW, which means more $\mathrm{THz}$ pulse energy and smaller bandwidth. Some working points can be identified with the help of a parametric study as shown in Fig. 8 .

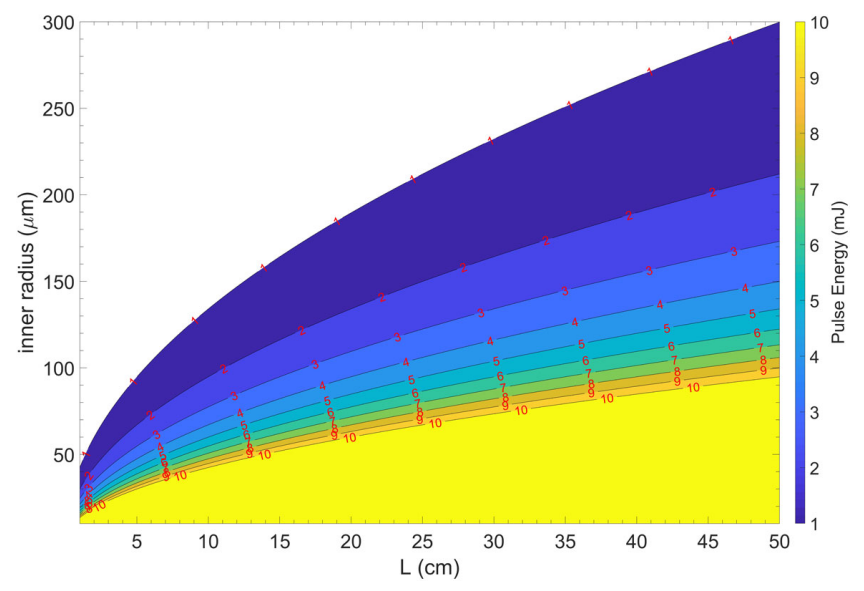

FIG. 6. Pulse energy dependence on $a$ and $L$ for a point charge of $100 \mathrm{pC}$. 


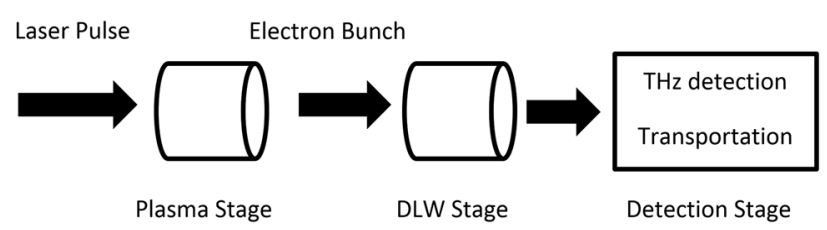

FIG. 7. Schematic of $\mathrm{THz}$ generation without magnetic capture and transport lines.

It is obvious that for the same pulse energy, a shorter DLW will accept a more divergent beam. There are operational points for beam divergence between 0.1 and $5 \mathrm{mrad}$ in order to generate $\mathrm{mJ}$ pulses. For example, an electron beam with $1 \mathrm{mrad}$ divergence requires a $2 \mathrm{~cm}$ long DLW with $20 \mu \mathrm{m}$ radius to generate $10 \mathrm{~mJ} \mathrm{THz}$ pulse and all the regions below the dashed line provide corresponding working points (see Fig. 8). Coupled with Fig. 3, it is possible to identify certain frequencies where $\mathrm{THz}$ pulses with energy on the order of a $\mathrm{mJ}$ or more are possible. It is tempting to use a shorter DLW with a small inner radius. However, the breakdown threshold of the dielectric material sets the upper limit one can operate in reality. For example, the inner radius will be limited approximately to $10 \mu \mathrm{m}$ to support $10 \mathrm{GV} / \mathrm{m}$ decelerating gradient. On the other hand, if the length of DLW is small compared with the free wavelength of the target $\mathrm{THz}$, the mode frequency will shift significantly if one starts to take into account the finite length effect. The desirable less than $10 \%$ narrow bandwidth also requires the DLW longer than one $\mathrm{cm}$ or so. Though it is an ultracompact solution for $\mathrm{THz}$ generation, it loses certain flexibility. One also faces no room for beam diagnostics. By transporting the electron beam from the plasma section to the DLW section, one can obtain more space.

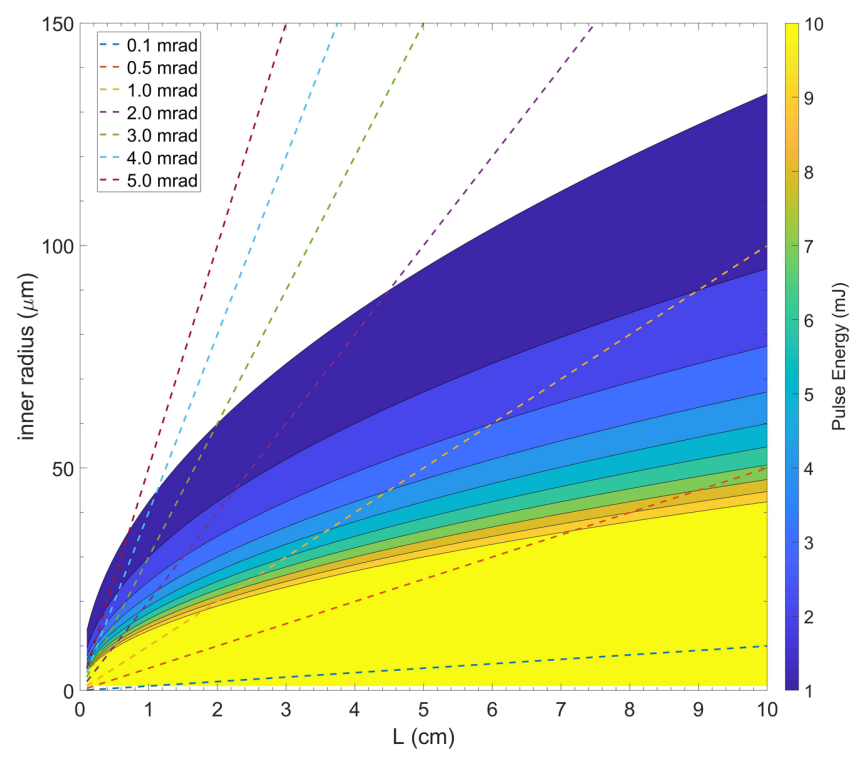

FIG. 8. Operational regions for different beam divergences.

\section{B. Capture and transport with magnetic line}

Since the electron beam from the laser plasma injector has a large divergence that limits the potential DLW length, it is necessary to correct the beam divergence immediately after the electron beam exits the plasma. It is almost impossible to match the beam with current magnet technology due to the mismatch of more than two orders of magnitude focusing strength. One option would be to use the plasma lens (magnetic quadrupole equivalent but with higher focusing strength) [48,49]. Another solution is to tailor the plasma density gradient so that the electron beam sees the plasma-vacuum interface gradually rather than abruptly. Although both approaches are attractive, they are still active research areas. Here we adopt the parametric study in [50] and consider the use of the conventional magnets based capture and transfer line.

Based on the published laser plasma acceleration experiments, we adopt the electron beam parameters in Table I. Electron beam with $\mathrm{GeV}$ energy, a few tens $\mathrm{pC}$ charge and sub mrad divergence can be generated experimentally [51]. But this would require a PW scale laser. The hundred $\mathrm{MeV}$ range we consider here should be feasible with commercial TW scale laser with few tens fs pulse length. The electron beam parameters are summarized in Table II for further study.

The transverse beam size expands quickly over the transport distance due to the mrad divergence of a typical beam obtained from a laser plasma accelerating process, which will in turn cause beam losses and affect the $\mathrm{THz}$ pulse energy. The normalized emittance $\varepsilon_{n, r m s}$ growth over a drift distance $s$ is [52]

$$
\varepsilon_{n, r m s}^{2}=\langle\gamma\rangle^{2}\left(\varepsilon_{0}^{2}+s^{2} \sigma_{E}^{2} \sigma_{x^{\prime}}^{4}\right)
$$

where $\varepsilon_{0}$ is the initial emittance, $\sigma_{E}$ and $\sigma_{x^{\prime}}$ beam energy spread and divergence, respectively. For energy spread on the order of a percent and mrad divergence, the chromatic emittance is added quadratically to the initial emittance $\varepsilon_{0}$. The chromatic emittance has strong dependence on the initial divergence $\sigma_{x^{\prime}}$ and energy spread $\sigma_{E}$. Its contribution quickly dominates the initial emittance $\varepsilon_{0}$. The strategy is therefore to provide a strong focusing on both transverse planes with a strong quadrupole triplet to control the beam size. In order to find out the working points in terms of beam divergence and energy spread, which are two parameters that have

TABLE II. Electron beam parameters for beam capture.

\begin{tabular}{lccc}
\hline \hline Parameter & Symbol & Value & Unit \\
\hline Beam Energy & $\gamma_{0} m c^{2}$ & 350 & $\mathrm{MeV}$ \\
RMS Energy spread & $\sigma_{\delta}$ & $\leq 5 \%$ & $/$ \\
RMS Beam size & $\sigma_{x, y}$ & $\leq 5$ & $(\mu \mathrm{m})$ \\
RMS Beam Divergence & $\sigma_{x, y}^{\prime}$ & $\leq 5$ & $\mathrm{mrad}$ \\
Charge & $Q$ & 100 & $\mathrm{pC}$ \\
RMS Bunch Length & $L_{s}$ & 10 & $\mathrm{fs}$ \\
Normalized Emittance & $\varepsilon_{n, r m s}$ & $\leq 5$ & $\mathrm{~mm} \mathrm{mrad}$ \\
\hline \hline
\end{tabular}




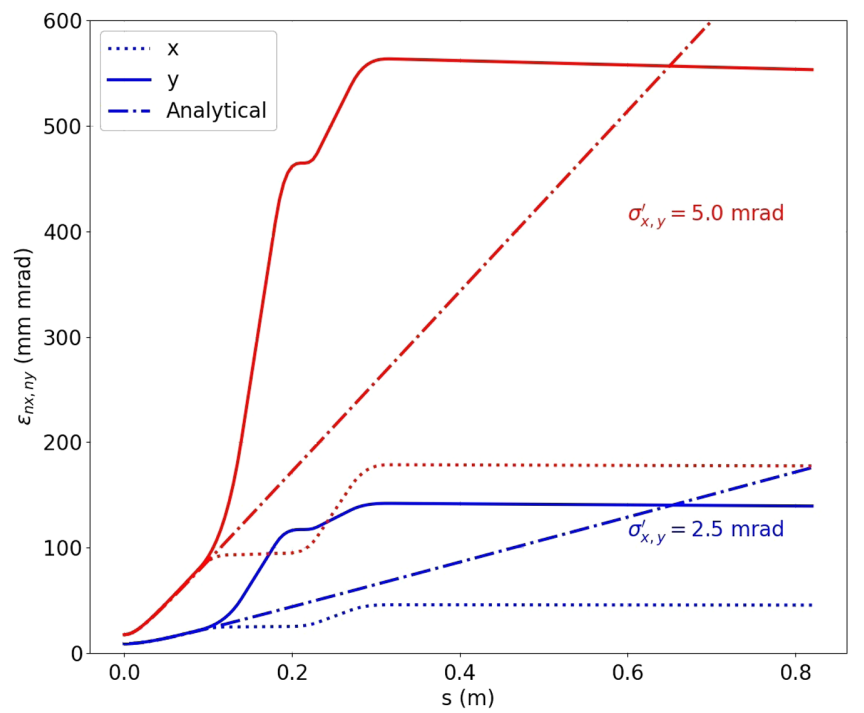

FIG. 9. Normalized emittance evolution along the capture triplet [dotted lines for $x$, solid lines for $y$ and dash-dotted for Eq. (4)]. The triplet is located at $0.1 \mathrm{~m}$ away from the plasma injector exit.

significant impact on beam quality evolution as indicated in Eq. (4), we have varied the initial energy spread between 0 and $5 \%$ and the divergence from 0 to $5 \mathrm{mrad}$. An optimized triplet as in Ref. [50] was used to show the normalized emittance $\varepsilon_{n, r m s}$ growth as the transport distance increases in Fig. 9 with a energy spread of $5 \%$.

It is obvious that the emittance grows quickly as the divergence increases. The triplet helps mitigate the emittance growth but it is still experiencing a quick growth. However, from the interaction we described above, we care more about the beam transverse size. The RMS beam sizes at different beam divergences are shown in Fig. 10.

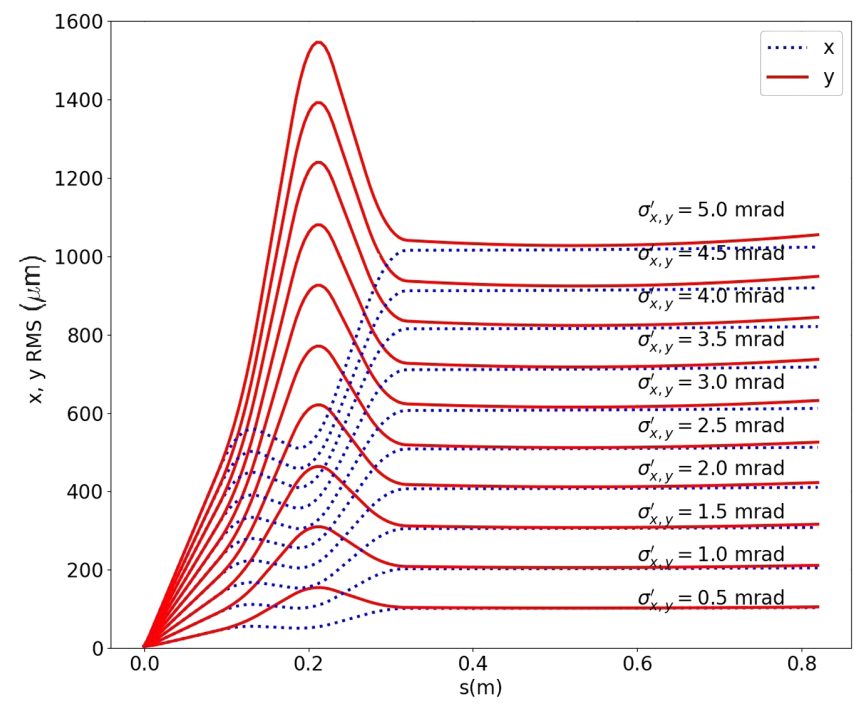

FIG. 10. Transverse beam sizes for various beam divergences over distance with a triplet.

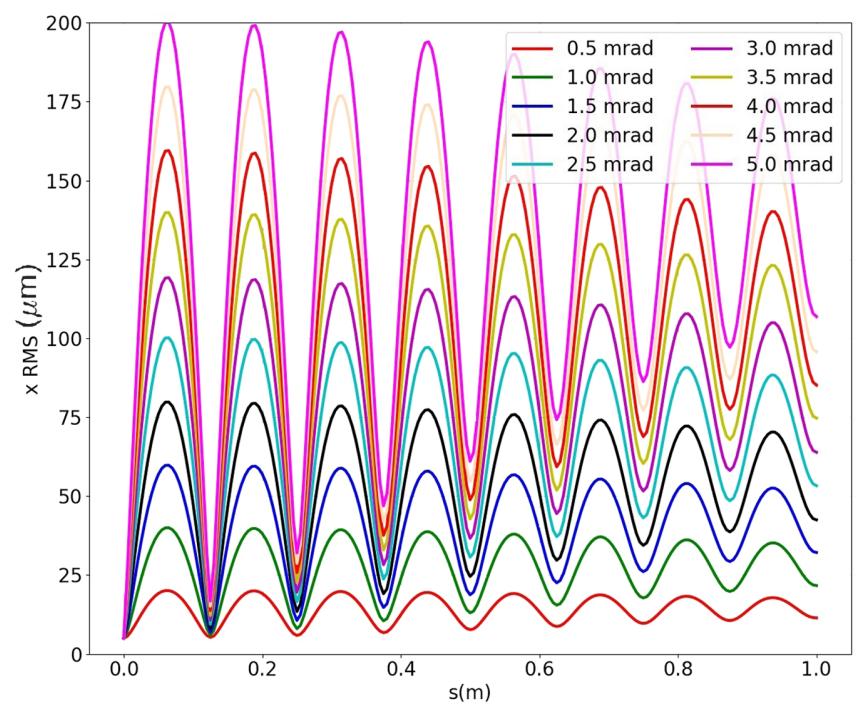

FIG. 11. Transverse beam size over distance with a solenoid for different beam divergences. Due to symmetry, only the beam size in $\mathrm{x}$ is shown and exactly the same results apply to $\mathrm{y}$ direction.

The triplet exit was located at $s=0.36 \mathrm{~m}$ and a $20-\mathrm{cm}$ long DLW can be placed next to it. It is still very critical to control the initial beam divergence in order to match the beam size to the small DLW. Even more complicated schemes such as double triplet or achromatic lines provide few benefits but add great costs and complexity to the initial experiments as concluded in Ref. [50].

A drift space of $10 \mathrm{~cm}$ between the exit of the plasma channel and the entrance of the triplet is introduced in order to make the focusing efficient. Due to this space, the beam size already blows up to approximately $100 \mu \mathrm{m}$ for a divergence of $1 \mathrm{mrad}$. In order to eliminate this size growth, we also considered the use of a magnetic solenoid to capture the divergent electron beam. With approximately $50 \mathrm{~T}$ central magnetic field, the transverse beam sizes are shown in Fig. 11 for different beam divergences up to 5 mrad with $5 \%$ energy spread.

The solenoid can be applied directly to the plasma exit so that the beam is focused in both directions simultaneously unlike the quadrupole focusing magnets. Though this capture method seems viable, the quest for the high field magnet does not disappear. For a beam with $1 \mathrm{mrad}$ divergence, the magnetic field strength $\mathrm{B}$ was varied to show the dependence in Fig. 12.

A moderate strength of $25 \mathrm{~T}$ should ensure a DLW of 1 meter, which can provide us above $10 \mathrm{~mJ} \mathrm{THz}$ pulse. This can host a longer DLW compared with the triplet setup. The $\mathrm{x}$ and $\mathrm{y}$ correlation introduced by solenoid is not a problem for us because of the azimuthal symmetry of the monopole modes. This fact on the contrary will help us mitigate the transverse kicks due to wakefield as we will see in next section.

A suitable working point requires a certain trade-off between the energy spread and divergence. Any improvement 


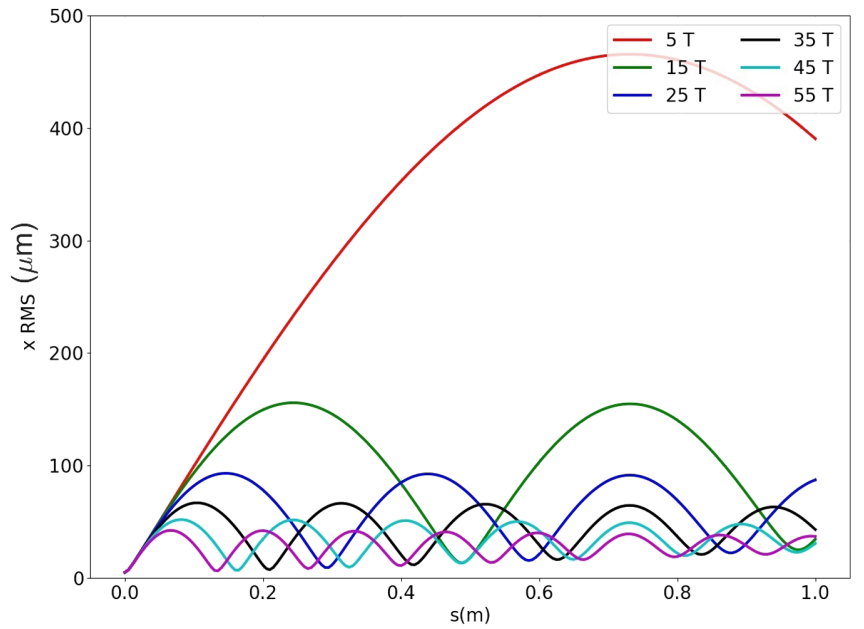

FIG. 12. Transverse beam size over distance with a solenoid for different magnetic field strength $\mathrm{B}$.

with lower divergence, high beam charge and less energy spread will improve the $\mathrm{THz}$ pulse energy. Besides the external focusing by various magnets, the electron bunch traveling inside the DLW also experiences transverse kicks from its self-excited field which is the topic for next section.

\section{BEAM BREAKUP DUE TO WAKEFIELD}

High deceleration gradient inside a DLW accompanies a strong transverse deflecting force based on the Panofsky and Wenzel theorem [53]. This causes shear displacements of the bunch and can lead to the head-to-tail instability, which might result in the electron beam loss in the DLW wall. For a well-aligned DLW with respect to the electron beam, only monopoles can be excited and the longitudinal force is [43],

$$
\left.F_{z}\right|_{l=0}=-2 q q_{0} \sum_{n=1}^{\infty} \frac{1}{C_{n 0}} \cos k_{n} s \Theta(-s)
$$

where $C_{n 0}$ is a normalization constant, related to loss factor defined in Eq. (3) as $\kappa_{n}=2 / C_{n}$, for monopole modes depending the DLW parameters, $q$ and $q_{0}$ are the leading and trailing charge, $k_{n}$ the wave vector for mode $n$. There is no transverse force contribution from this mode up to the order of $\gamma^{-2}$ due to the cancellation of the transverse force from the magnetic and electric field. If the DLW is slightly misaligned transversely, dipole, quadrupole modes can be excited and the transverse kick is dominated by the dipole modes [43],

$$
\begin{gathered}
\left.F_{x}(x, y, s)\right|_{l=1}=-4 q q_{0} \frac{r_{0}}{R_{1}} \sum_{n=1}^{\infty} \frac{1}{C_{n 1}} \frac{\sin k_{n} s}{k_{n} R_{1}} \Theta(-s) \\
\text { and }\left.\quad F_{y}(x, y, s)\right|_{l=1}=0 .
\end{gathered}
$$

The transverse kick at the trailing particle depends on the transverse offset $r_{0}$ of the preceding particle. When the preceding particle is horizontally $(x)$ displaced, the kick in the $y$ direction is zero and this fact also favors the use of solenoid because of the $x-y$ coupling introduced. In the following, we consider one extreme situation where the particles are solely concentrated on the $x-s$ plane. The particle distribution at different location along $s$ is shown in Fig. 13. The bunch starts with a slight offset of $1 \mu \mathrm{m}$ with zero divergence. We gradually see the transverse kick to the bunch tail and finally a particle loss in the dielectric wall after $60 \mathrm{~cm}$.

Upon the trailing particle transverse offset being negative or positive, it can be either decelerated or accelerated. This will further add to the energy spread of the bunch. The transverse force is generally one order of magnitude smaller than the longitudinal force if one assumes percent level misalignment.
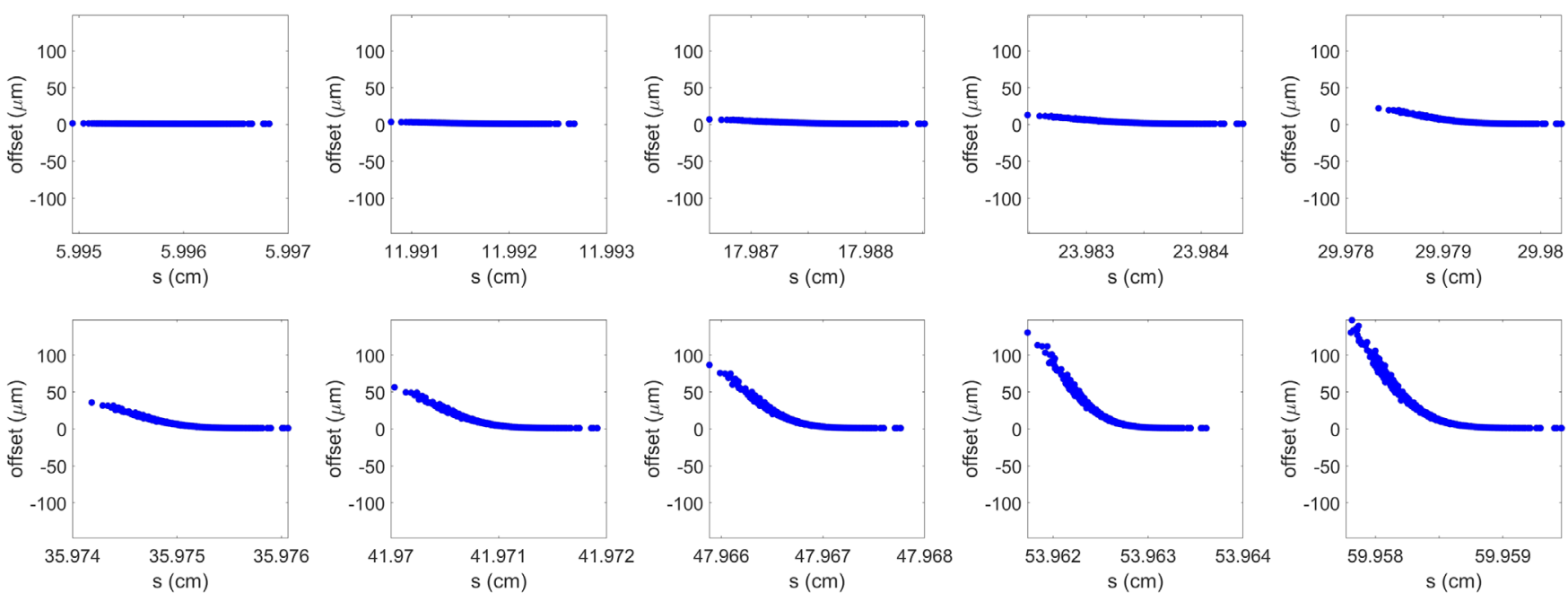

FIG. 13. Snapshot of beam movement, which offsets $1 \mu \mathrm{m}$, inside a DLW ( $a=148 \mu \mathrm{m}$ and $b=178 \mu \mathrm{m})$ at different location $s$. 
It is obvious that the transverse kicks can be mitigated by centering the electron beam with respect to the axis of a DLW, which can be positioned on a hexpod like platform with six degrees of freedom. This allows us to correct not only the transverse misalignment but also the tilt of the DLW with respect to the electron beam. A beam-based alignment can be conducted therein.

Although the $\mathrm{mJ}$ per pulse energy is already at least one order of magnitude higher than what one can obtain from an undulator based approach, which is limited by the electron to photon energy conversion rate, it is possible to further enhance the photon yield by manipulating the electron beam longitudinal structure. This is essentially to change the form factor of a certain bunch so that certain $\mathrm{TM}_{0 \mathrm{n}}$ mode is resonantly excited. Due to the inherent energy chirp of the plasma accelerated electron bunch, the optics can be hosted in the beam transport line with a dog leg as in [54] after the proof of principle experiments.

\section{POTENTIAL EXPERIMENTAL APPLICATIONS}

Intense narrowband $\mathrm{THz}$-frequency light pulses with a few percent bandwidth have several interesting potential applications in solid-state physics. Many such applications rest on the idea of using the pulses to drive vibrational resonances. These typically lie in the $1-30 \mathrm{THz}$ range, and have line-widths ranging from $1 \%-40 \%$ bandwidth. Recently there have been several experiments showing that focused midinfrared and $\mathrm{THz}$-frequency light can drive large-scale atomic motions that can drive changes in the properties of the solid via linear [55] or nonlinear couplings [56-63]. An example of the latter case includes the observation of transient superconducting like behavior in several different systems driven by multi- $\mathrm{THz}$ frequency light in resonance with vibrational modes [57,59-62]. The mechanisms for these observations are not well understood and have stimulated new theoretical efforts in understanding nonequilibrium superconductivity [64-67]. Nonlinear phonon-phonon interactions have also been proposed as a way to drive transient multiferroicity in nonmagnetic materials via resonant enhancement of a time-dependent polarization [63]. The nonlinear coupling of vibrational coordinates to electronic states have also been proposed to drive modulations of conductivity that in some cases result in metallization of insulators on ultrafast timescales [68].

In addition to applications involving the resonant driving of vibrations, there is also some burgeoning interest in using intense $\mathrm{THz}$ pulses and their interactions with electronic degrees of freedom to study Floquet physics in solid-state materials. Many-body systems with strong periodic driving are predicted to have lowest energy states with properties that are quite different from those obtainable in equilibrium $[69,70]$. Examples of somewhat surprising phenomena that have been predicted include dynamic localization [71,72], dynamically driven topological phase transitions [73-76] and spontaneous symmetry breaking [77]. Some of these theoretical predictions have been validated in cold atom systems [78] and in photonic waveguides [79], and also in a few experiments on condensed matter systems $[80,81]$. This points to the exciting possibility of using periodic driving as a means of control in solid-state materials, an idea often referred to as "Floquet engineering." One main difficulty in practical realization of Floquet engineering is the problem of heating: dissipation channels limit the length of time over which one can apply strong periodic driving without heating up the material to very high levels [82]. Theoretical work has shown that in many systems there exists a period of "pre-thermalization," when the effects of heating are not yet severe but the system has otherwise settled into a steady state. In many systems with electronic band gaps on the order of $1 \mathrm{eV}$, this regime may be realized for intense 1\%-10\% bandwidth light pulses in the THz-frequency range.

In order to understand better the novel material states that could be created by the $\mathrm{THz}$ interaction, there must be a way to measure the properties of these states on timescales before thermalizaiton takes place. Ultrashort x-ray pulses from a FEL are in many ways ideal probes that offer selective measurements of different aspects of the structure of these transient states. X-ray scattering, for example, can be used to create snapshots of the electron density distribution with femtosecond time resolution [83,84]. By tuning the $\mathrm{x}$-ray wavelength to core level resonances it also becomes possible to measure the structure of spin and other valence electron properties with atomic resolution $[55,85,86]$. The possibilities for combining the $\mathrm{THz}$ source discussed here with the full capabilities of an x-ray FEL offer unique opportunities for understanding the properties of condensed matter under conditions of strong driving by a periodic electromagnetic field.

\section{SUMMARY AND DISCUSSION}

We have proposed a possible narrowband and powerful $\mathrm{THz}$ generation method for the SwissFEL beamlines where the users can perform $\mathrm{THz}$ pump and $\mathrm{x}$-ray probe experiments, which can completely push the potential experiments to a new era. The method utilizes an external electron source coming from a laser plasma injector and a dielectric lined waveguide. The investigation forms a compact solution for the high energy $\mathrm{THz}$ generation by benefiting from the research of laser plasma acceleration and dielectric high gradient structure.

With the typical LPW electron beam parameters, we could generate $\mathrm{mJ} \mathrm{THz}$ pulses. Due to the mrad divergence, the electron beam capture with magnetic line and transport through the DLW are still challenging but feasible. We found three working regions: 1 . use small radius DLW to generate $\mathrm{THz}$ pulse before the electron beam hits the DLW wall 2. utilize the high gradient PMQs to mitigate the divergence effects and this allows us a few tens of cm DLW 3. capture the divergent beam with a solenoid. The use of 
solenoid seems the best option since it provides focusing for both transverse planes at the same time. A sub-meter DLW can be used inside the solenoid and this also guarantees the narrow bandwidth to meet user's needs.

The laser used for LPW is intrinsically synchronized with the SwissFEL linac and thus the $\mathrm{THz}$ pulses are synchronized with the x-ray pulses. This enables users to perform a wide range of pump-probe experiments, where timing is of paramount important.

The study here can be implemented independently as a narrowband high pulse energy $\mathrm{THz}$ source as well. It should be noted that LPW is a rapidly developing area and with all the state-of-the-art experimental conditions, it is feasible physically to produce multiple $\mathrm{GeV}$ electron beam with sub $\mathrm{nC}$ charge and sub mrad divergence. This will definitely push the energy frontier of the $\mathrm{THz}$ pulse generation.

\section{ACKNOWLEDGMENTS}

We wish to acknowledge the discussion with Sergey Tomin for the OCELOT package used for the parametric optics study. We also thank Antici Patrizio for the laser plasma discussion. This project has received funding from the European Unions Horizon 2020 research and innovation programme under the Marie Skodowska-Curie Grant Agreement No. 701647. This research was supported by the NCCR MUST, funded by the Swiss National Science Foundation.

[1] T. Kampfrath, K. Tanaka, and K. A. Nelson, Resonant and nonresonant control over matter and light by intense terahertz transients, Nat. Photonics 7, 680 (2013).

[2] J.-M. Manceau, P. A. Loukakos, and S. Tzortzakis, Direct acoustic phonon excitation by intense and ultrashort terahertz pulses, Appl. Phys. Lett. 97, 251904 (2010).

[3] C. Milne, T. Schietinger, M. Aiba, A. Alarcon, J. Alex, A. Anghel, V. Arsov, C. Beard, P. Beaud, S. Bettoni et al., SwissFEL: The swiss X-ray free electron laser, Appl. Sci. 7, 720 (2017).

[4] S. Kovalev, B. Green, T. Golz, S. Maehrlein, N. Stojanovic, A. Fisher, T. Kampfrath, and M. Gensch, Probing ultra-fast processes with high dynamic range at 4th-generation light sources: Arrival time and intensity binning at unprecedented repetition rates, Structural Dynamics 4, 024301 (2017).

[5] S. Dhillon, M. Vitiello, E. Linfield, A. Davies, M. C. Hoffmann, J. Booske, C. Paoloni, M. Gensch, P. Weightman, G. Williams et al., The 2017 terahertz science and technology roadmap, J. Phys. D 50, 043001 (2017).

[6] R. A. Lewis, A review of terahertz sources, J. Phys. D 47, 374001 (2014).

[7] S. Pérez, T. González, D. Pardo, and J. Mateos, Terahertz Gunn-like oscillations in InGaAs/InAlAs planar diodes, J. Appl. Phys. 103, 094516 (2008).
[8] K. B. Kwon, T. Kang, H. S. Song, Y.-K. Kim, B. Ersfeld, D. A. Jaroszynski, and M. S. Hur, High-energy, shortduration bursts of coherent terahertz radiation from an embedded plasma dipole, Sci. Rep. 8, 145 (2018).

[9] B.S. Williams, Terahertz quantum-cascade lasers, Nat. Photonics 1, 517 (2007).

[10] H. Hübers, S. Pavlov, and V. Shastin, Terahertz lasers based on germanium and silicon, Semicond. Sci. Technol. 20, S211 (2005).

[11] S. Antipov, C. Jing, P. Schoessow, A. Kanareykin, V. Yakimenko, A. Zholents, and W. Gai, High power terahertz radiation source based on electron beam wakefields, Rev. Sci. Instrum. 84, 022706 (2013).

[12] W. Leemans, C. Geddes, J. Faure, C. Tóth, J. Van Tilborg, C. Schroeder, E. Esarey, G. Fubiani, D. Auerbach, B. Marcelis et al., Observation of Terahertz Emission from a LaserPlasma Accelerated Electron Bunch Crossing a PlasmaVacuum Boundary, Phys. Rev. Lett. 91, 074802 (2003).

[13] L. Shi, S. Bettoni, M. Dehler, E. Ferrari, B. Hermann, R. Ischebeck, F. Marcellini, A. Mittelbach, S. Reiche, and V. Thominet, in 29th Linear Accelerator Conf.(LINAC'18), Beijing, China, 16-21 September 2018 (JACOW Publishing, Geneva, Switzerland, 2018) pp. 94-97.

[14] A. D. Cahill, J. B. Rosenzweig, V. A. Dolgashev, S. G. Tantawi, and S. Weathersby, High gradient experiments with X -band cryogenic copper accelerating cavities, Phys. Rev. Accel. Beams 21, 102002 (2018).

[15] R. Geng, Review of new shapes for higher gradients, Physica C (Amsterdam) 441, 145 (2006).

[16] R. Geng et al., in the Proceedings of SRF2011, Chicago, Illinois, USA (2011), p. 74, http://accelconf.web.cern.ch/ AccelConf/SRF2011/papers/mopo012.pdf.

[17] H. Padamsee, J. Knobloch, T. Hays et al., RF Superconductivity for Accelerators, Vol. 2011 (Wiley Online Library, New York, 2008).

[18] T. Tajima and J. M. Dawson, Laser Electron Accelerator, Phys. Rev. Lett. 43, 267 (1979).

[19] J. Faure, Y. Glinec, A. Pukhov, S. Kiselev, S. Gordienko, E. Lefebvre, J.-P. Rousseau, F. Burgy, and V. Malka, A laserplasma accelerator producing monoenergetic electron beams, Nature (London) 431, 541 (2004).

[20] C. Geddes, C. Toth, J. Van Tilborg, E. Esarey, C. Schroeder, D. Bruhwiler, C. Nieter, J. Cary, and W. Leemans, High-quality electron beams from a laser wakefield accelerator using plasma-channel guiding, Nature (London) 431, 538 (2004).

[21] S. P. Mangles, C. Murphy, Z. Najmudin, A. G. R. Thomas, J. Collier, A. E. Dangor, E. Divall, P. Foster, J. Gallacher, C. Hooker et al., Monoenergetic beams of relativistic electrons from intense laser-plasma interactions, Nature (London) 431, 535 (2004).

[22] D. Strickland and G. Mourou, Compression of amplified chirped optical pulses, Opt. Commun. 55, 447 (1985).

[23] W. Li, Z. He, Y. Lu, R. Huang, W. Liu, Q. Jia, and L. Wang, Tunable $\mathrm{THz}$ radiation source from dielectric loaded waveguide excited by nonrelativistic electron bunch trains, Phys. Rev. Accel. Beams 19, 104701 (2016).

[24] A. Cook, R. Tikhoplav, S. Y. Tochitsky, G. Travish, O. Williams, and J. Rosenzweig, Observation of NarrowBand Terahertz Coherent Cherenkov Radiation from a 
Cylindrical Dielectric-Lined Waveguide, Phys. Rev. Lett. 103, 095003 (2009).

[25] S. Antipov, S. Baryshev, R. Kostin, S. Baturin, J. Qiu, C. Jing, C. Swinson, M. Fedurin, and D. Wang, Efficient extraction of high power $\mathrm{THz}$ radiation generated by an ultra-relativistic electron beam in a dielectric loaded waveguide, Appl. Phys. Lett. 109, 142901 (2016).

[26] S. Antipov, M. Babzien, C. Jing, M. Fedurin, W. Gai, A. Kanareykin, K. Kusche, V. Yakimenko, and A. Zholents, Subpicosecond Bunch Train Production for a Tunable mJ Level THz Source, Phys. Rev. Lett. 111, 134802 (2013).

[27] E. Esarey, C. Schroeder, and W. Leemans, Physics of laser-driven plasma-based electron accelerators, Rev. Mod. Phys. 81, 1229 (2009).

[28] H. Ekerfelt, M. Hansson, I. G. González, X. Davoine, and O. Lundh, A tunable electron beam source using trapping of electrons in a density down-ramp in laser wakefield acceleration, Sci. Rep. 7, 12229 (2017).

[29] S. Bulanov, F. Pegoraro, A. Pukhov, and A. Sakharov, Transverse-Wake Wave Breaking, Phys. Rev. Lett. 78, 4205 (1997).

[30] A. Pak, K. Marsh, S. Martins, W. Lu, W. Mori, and C. Joshi, Injection and Trapping of Tunnel-Ionized Electrons into Laser-Produced Wakes, Phys. Rev. Lett. 104, 025003 (2010).

[31] S. Bulanov, N. Naumova, F. Pegoraro, and J. Sakai, Particle injection into the wave acceleration phase due to nonlinear wake wave breaking, Phys. Rev. E 58, R5257 (1998).

[32] A. F. Pousa, R. Assmann, and A. M. de la Ossa, in 9th International Particle Accelerator Conference (IPAC2018), Vancouver, Canada, April 2018 (2018), pp. 607-611, https://accelconf.web.cern.ch/AccelConf/ipac2018/papers/ tuxgbe4.pdf.

[33] D. Middleton, Ultra-high frequency oscillations of cylindrical cavity resonators containing two and three dielectric media, Phys. Rev. 63, 343 (1943).

[34] M. Rosing and W. Gai, Longitudinal- and transverse-wakefield effects in dielectric structures, Phys. Rev. D 42, 1829 (1990).

[35] T.-B. Zhang, J. L. Hirshfield, T. C. Marshall, and B. Hafizi, Stimulated dielectric wake-field accelerator, Phys. Rev. E 56, 4647 (1997).

[36] J. G. Power, M. E. Conde, W. Gai, R. Konecny, P. Schoessow, and A. D. Kanareykin, Measurements of the longitudinal wakefields in a multimode, dielectric wakefield accelerator driven by a train of electron bunches, Phys. Rev. Accel. Beams 3, 101302 (2000).

[37] G. V. Sotnikov, T. C. Marshall, and J. L. Hirshfield, Coaxial two-channel high-gradient dielectric wakefield accelerator, Phys. Rev. Accel. Beams 12, 061302 (2009).

[38] G. Andonian, O. Williams, S. Barber, D. Bruhwiler, P. Favier, M. Fedurin, K. Fitzmorris, A. Fukasawa, P. Hoang, K. Kusche, B. Naranjo, B. O'Shea, P. Stoltz, C. Swinson, A. Valloni, and J. B. Rosenzweig, Planar-DielectricWakefield Accelerator Structure Using Bragg-Reflector Boundaries, Phys. Rev. Lett. 113, 264801 (2014).

[39] A. Hanuka and L. Schächter, Optimized operation of dielectric laser accelerators: Single bunch, Phys. Rev. Accel. Beams 21, 054001 (2018).
[40] F. Gao, M. Conde, W. Gai, C. Jing, R. Konecny, W. Liu, J. Power, T. Wong, and Z. Yusof, Design and testing of a $7.8 \mathrm{GHz}$ power extractor using a cylindrical dielectricloaded waveguide, Phys. Rev. Accel. Beams 11, 041301 (2008).

[41] A. Altmark and A. Kanareykin, in Journal of Physics: Conference Series, Vol. 732 (IOP Publishing, London, 2016), p. 012037.

[42] K.-Y. Ng, Wake fields in a dielectric-lined waveguide, Phys. Rev. D 42, 1819 (1990).

[43] S. Park and J. Hirshfield, Theory of wakefields in a dielectric-lined waveguide, Phys. Rev. E 62, 1266 (2000).

[44] L. Schächter, R. Byer, and R. Siemann, Wake field in dielectric acceleration structures, Phys. Rev. E 68, 036502 (2003).

[45] S. Baturin and A. Kanareykin, New method of calculating the wakefields of a point charge in a waveguide of arbitrary cross section, Phys. Rev. Accel. Beams 19, 051001 (2016).

[46] S. Antipov and L. Yan (private communication).

[47] M. C. Thompson, H. Badakov, A. M. Cook, J. B. Rosenzweig, R. Tikhoplav, G. Travish, I. Blumenfeld, M. J. Hogan, R. Ischebeck, N. Kirby, R. Siemann, D. Walz, P. Muggli, A. Scott, and R. B. Yoder, Breakdown Limits on Gigavolt-per-Meter Electron-Beam-Driven Wakefields in Dielectric Structures, Phys. Rev. Lett. 100, 214801 (2008).

[48] C. Thaury, E. Guillaume, A. Döpp, R. Lehe, A. Lifschitz, K. T. Phuoc, J. Gautier, J.-P. Goddet, A. Tafzi, A. Flacco et al., Demonstration of relativistic electron beam focusing by a laser-plasma lens, Nat. Commun. 6, 6860 (2015).

[49] R. Lehe, C. Thaury, E. Guillaume, A. Lifschitz, and V. Malka, Laser-plasma lens for laser-wakefield accelerators, Phys. Rev. Accel. Beams 17, 121301 (2014).

[50] M. Scisciò, L. Lancia, M. Migliorati, A. Mostacci, L. Palumbo, Y. Papaphilippou, and P. Antici, Parametric study of transport beam lines for electron beams accelerated by laser-plasma interaction, J. Appl. Phys. 119, 094905 (2016).

[51] W. P. Leemans, A. J. Gonsalves, H.-S. Mao, K. Nakamura, C. Benedetti, C. B. Schroeder, C. Tóth, J. Daniels, D. E. Mittelberger, S. S. Bulanov, J.-L. Vay, C. G. R. Geddes, and E. Esarey, Multi-GeV Electron Beams from CapillaryDischarge-Guided Subpetawatt Laser Pulses in the SelfTrapping Regime, Phys. Rev. Lett. 113, 245002 (2014).

[52] M. Migliorati, A. Bacci, C. Benedetti, E. Chiadroni, M. Ferrario, A. Mostacci, L. Palumbo, A. R. Rossi, L. Serafini, and P. Antici, Intrinsic normalized emittance growth in laser-driven electron accelerators, Phys. Rev. Accel. Beams 16, 011302 (2013).

[53] W. Panofsky and W. Wenzel, Some considerations concerning the transverse deflection of charged particles in radio-frequency fields, Rev. Sci. Instrum. 27, 967 (1956).

[54] P. Muggli, V. Yakimenko, M. Babzien, E. Kallos, and K. Kusche, Generation of Trains of Electron Microbunches with Adjustable Subpicosecond Spacing, Phys. Rev. Lett. 101, 054801 (2008).

[55] T. Kubacka, J. A. Johnson, M. C. Hoffmann, C. Vicario, S. De Jong, P. Beaud, S. Grübel, S.-W. Huang, L. Huber, L. Patthey et al., Large-amplitude spin dynamics driven by a $\mathrm{THz}$ pulse in resonance with an electromagnon, Science 343, 1333 (2014). 
[56] M. Rini, N. Dean, J. Itatani, Y. Tomioka, Y. Tokura, R. W. Schoenlein, A. Cavalleri, Control of the electronic phase of a manganite by mode-selective vibrational excitation, Nature (London) 449, 72 (2007).

[57] D. Fausti, R. Tobey, N. Dean, S. Kaiser, A. Dienst, M. C. Hoffmann, S. Pyon, T. Takayama, H. Takagi, and A. Cavalleri, Light-induced superconductivity in a stripeordered cuprate, Science 331, 189 (2011).

[58] M. Först, C. Manzoni, S. Kaiser, Y. Tomioka, Y. Tokura, R. Merlin, and A. Cavalleri, Nonlinear phononics as an ultrafast route to lattice control, Nat. Phys. 7, 854 (2011).

[59] R. Mankowsky, A. Subedi, M. Först, S. O. Mariager, M. Chollet, H. Lemke, J. S. Robinson, J. M. Glownia, M. P. Minitti, A. Frano et al., Nonlinear lattice dynamics as a basis for enhanced superconductivity in $\mathrm{YBa}_{2} \mathrm{Cu}_{3} \mathrm{O}_{6.5}$, Nature (London) 516, 71 (2014).

[60] W. Hu, S. Kaiser, D. Nicoletti, C. R. Hunt, I. Gierz, M. C. Hoffmann, M. Le Tacon, T. Loew, B. Keimer, and A. Cavalleri, Optically enhanced coherent transport in $\mathrm{YBa}_{2} \mathrm{Cu}_{3} \mathrm{O}_{6.5}$ by ultrafast redistribution of interlayer coupling, Nat. Mater. 13, 705 (2014).

[61] A. Cantaluppi, M. Buzzi, G. Jotzu, D. Nicoletti, M. Mitrano, D. Pontiroli, M. Riccò, A. Perucchi, P. Di Pietro, and A. Cavalleri, Pressure tuning of light-induced superconductivity in $K_{3} C_{60}$, Nat. Phys. 14, 837 (2018).

[62] M. Mitrano, A. Cantaluppi, D. Nicoletti, S. Kaiser, A. Perucchi, S. Lupi, P. Di Pietro, D. Pontiroli, M. Riccò, S. R. Clark et al., Possible light-induced superconductivity in $K_{3} C_{60}$ at high temperature, Nature (London) 530, 461 (2016).

[63] D. M. Juraschek, M. Fechner, A. V. Balatsky, and N. A. Spaldin, Dynamical multiferroicity, Phys. Rev. Mater. 1, 014401 (2017).

[64] M. Babadi, M. Knap, I. Martin, G. Refael, and E. Demler, Theory of parametrically amplified electron-phonon superconductivity, Phys. Rev. B 96, 014512 (2017).

[65] M. Knap, M. Babadi, G. Refael, I. Martin, and E. Demler, Dynamical Cooper pairing in nonequilibrium electronphonon systems, Phys. Rev. B 94, 214504 (2016).

[66] D. M. Kennes, E. Y. Wilner, D. R. Reichman, and A. J. Millis, Transient superconductivity from electronic squeezing of optically pumped phonons, Nat. Phys. 13, 479 (2017).

[67] G. Chiriacò, A. J. Millis, and I. L. Aleiner, Transient superconductivity without superconductivity, Phys. Rev. B 98, 220510 (2018).

[68] V. Esposito, M. Fechner, R. Mankowsky, H. Lemke, M. Chollet, J. Glownia, M. Nakamura, M. Kawasaki, Y. Tokura, U. Staub et al., Nonlinear Electron-Phonon Coupling in Doped Manganites, Phys. Rev. Lett. 118, 247601 (2017).

[69] K. Sacha and J. Zakrzewski, Time crystals: A review, Rep. Prog. Phys. 81, 016401 (2017).

[70] P. Liang, M. Marthaler, and L. Guo, Floquet many-body engineering: Topology and many-body physics in phase space lattices, New J. Phys. 20, 023043 (2018).
[71] D. Dunlap and V. Kenkre, Dynamic localization of a charged particle moving under the influence of an electric field, Phys. Rev. B 34, 3625 (1986).

[72] N. Tsuji, T. Oka, and H. Aoki, Correlated electron systems periodically driven out of equilibrium: Floquet + DMFT formalism, Phys. Rev. B 78, 235124 (2008).

[73] T. Oka and H. Aoki, Photovoltaic Hall effect in graphene, Phys. Rev. B 79, 081406 (2009).

[74] N. H. Lindner, G. Refael, and V. Galitski, Floquet topological insulator in semiconductor quantum wells, Nat. Phys. 7, 490 (2011).

[75] T. Kitagawa, E. Berg, M. Rudner, and E. Demler, Topological characterization of periodically driven quantum systems, Phys. Rev. B 82, 235114 (2010).

[76] G. Usaj, P. Perez-Piskunow, L. F. Torres, and C. Balseiro, Irradiated graphene as a tunable Floquet topological insulator, Phys. Rev. B 90, 115423 (2014).

[77] M. S. Rudner and J. C. Song, Self-induced Berry flux and spontaneous non-equilibrium magnetism, Nat. Phys. 15, 1017 (2019).

[78] G. Jotzu, M. Messer, R. Desbuquois, M. Lebrat, T. Uehlinger, D. Greif, and T. Esslinger, Experimental realization of the topological Haldane model with ultracold fermions, Nature (London) 515, 237 (2014).

[79] M. C. Rechtsman, J. M. Zeuner, Y. Plotnik, Y. Lumer, D. Podolsky, F. Dreisow, S. Nolte, M. Segev, and A. Szameit, Photonic Floquet topological insulators, Nature (London) 496, 196 (2013).

[80] T. Ishikawa, Y. Sagae, Y. Naitoh, Y. Kawakami, H. Itoh, K. Yamamoto, K. Yakushi, H. Kishida, T. Sasaki, S. Ishihara et al., Optical freezing of charge motion in an organic conductor, Nat. Commun. 5, 5528 (2014).

[81] Y. Wang, H. Steinberg, P. Jarillo-Herrero, and N. Gedik, Observation of Floquet-Bloch states on the surface of a topological insulator, Science 342, 453 (2013).

[82] R. Moessner and S. L. Sondhi, Equilibration and order in quantum Floquet matter, Nat. Phys. 13, 424 (2017).

[83] R. A. Kirian and H. N. Chapman, in Synchrotron Light Sources and Free-Electron Lasers (Springer International Publishing, New York, 2015) pp. 1-55.

[84] C. Bostedt, S. Boutet, D. M. Fritz, Z. Huang, H. J. Lee, H. T. Lemke, A. Robert, W. F. Schlotter, J. J. Turner, and G. J. Williams, Linac coherent light source: The first five years, Rev. Mod. Phys. 88, 015007 (2016).

[85] S. Johnson, R. De Souza, U. Staub, P. Beaud, E. MöhrVorobeva, G. Ingold, A. Caviezel, V. Scagnoli, W. Schlotter, J. Turner et al., Femtosecond Dynamics of the Collinear-to-Spiral Antiferromagnetic Phase Transition in CuO, Phys. Rev. Lett. 108, 037203 (2012).

[86] P. Beaud, A. Caviezel, S. Mariager, L. Rettig, G. Ingold, C. Dornes, S. Huang, J. Johnson, M. Radovic, T. Huber et al., A time-dependent order parameter for ultrafast photoinduced phase transitions, Nat. Mater. 13, 923 (2014). 